\title{
Traduire
}

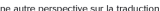

Revue française de la traduction

$229 \mid 2013$

L'environnement, une spécialisation durable?

\section{Le développement durable en traduction}

\section{Christine Cross}

\section{OpenEdition}

Journals

Édition électronique

URL : http://journals.openedition.org/traduire/577

DOI : 10.4000/traduire. 577

ISSN : 2272-9992

\section{Éditeur}

Société française des traducteurs

\section{Édition imprimée}

Date de publication : 15 décembre 2013

Pagination : 12-15

ISSN : 0395-773X

\section{Référence électronique}

Christine Cross, «Le développement durable en traduction », Traduire [En ligne], 229 | 2013, mis en

ligne le 15 décembre 2015, consulté le 10 décembre 2020. URL : http://journals.openedition.org/ traduire/577 ; DOI : https://doi.org/10.4000/traduire.577 


\section{Le développement durable en traduction}

\section{Christine Cross}

Dans un monde qui évolue à une vitesse vertigineuse, le traducteur se doit de suivre l'actualité de très près, quel que soit le domaine concerné. De nouveaux concepts et de nouvelles expressions apparaissent sans arrêt et gare à celui ou à celle qui tarde à les intégrer. Les thèmes qui aujourd'hui nous paraissent étranges et lointains seront probablement, dès demain, sur toutes les lèvres.

Ce phénomène est particulièrement marqué dans le domaine environnemental. Certains ont parfois du mal à se rappeler que l'écologie sous toutes ses formes, la défense et la protection de notre planète, le réchauffement climatique et la transition énergétique étaient autant de thèmes presque méconnus dans un passé relativement récent.

Le " développement durable ", quant à lui, a vu le jour dans les années 1990. C'est dans le cadre d'une conférence internationale dans le domaine du transport que j'ai entendu cette expression pour la première fois. J'ai eu la chance d'être en cabine anglaise lorsque l'un des intervenants a prononcé les mots sustainable development. De la chance, car ce sont mes collègues francophones et germanophones qui ont été sur la sellette. Quant à moi, dès ma sortie de cabine, je me suis dépêchée d'étudier le sujet pour être prête à l'affronter le jour où il recroiserait mon chemin. Et cela n'a pas tardé !

Ouvrons à ce stade une petite parenthèse : dans son article "Traduire dans le domaine de l'économie écologique : les difficultés terminologiques(1) ", Sabri-Fabrice Sayhi nous a expliqué que la traduction française "développement durable ", pourtant courante, est contestée dans certains milieux, le sens primitif de sustainable correspondant plutôt à la notion de " soutenabilité(2) ". Qui sait ? Le qualificatif " durable " doit peut-être son existence à un interprète qui, pris de court, a imaginé cette solution pour se tirer d'affaire. Mais là, je fabule un peu!

(1) Article paru dans le numéro 227 de Traduire "Éco, socio, philo \& co » p. 35-47.

(2) Voir aussi à ce sujet, dans le présent numéro, l'article de Jean-François Allain, "Durable. Vous avez dit durable ? " p. 8. 
Aujourd'hui le développement durable fait partie de notre univers de tous les jours. II est tellement omniprésent que même les traducteurs qui ne travaillent pas spécifiquement dans le domaine environnemental le retrouvent souvent dans les textes qu'on leur confie. Nombreuses, par exemple, sont les entreprises qui dans leur rapport annuel aux actionnaires, conformément à la législation en vigueur, font état de leurs gestes pour minimiser l'impact de leurs activités sur l'environnement et protéger la planète. Un grand distributeur détaillera ses efforts pour rationaliser, voire limiter, ses flux de transport et donc pour maîtriser ses nuisances et favoriser un développement équilibré et durable. Une entreprise de recyclage de déchets expliquera en quoi ses procédures sont propres et non polluantes. Même en dehors des obligations légales, tout le monde semble désireux de faire valoir des comportements écologiques(3).

Dans mon domaine de prédilection, qui est celui du transport ferroviaire, la carte écologique peut représenter un atout majeur. La performance énergétique du mode ferré est documentée depuis bien longtemps. Cela dit, le secteur est constamment en quête de nouvelles améliorations face à une concurrence qui fait preuve d'imagination et de talent dans ses efforts de réduction de son empreinte carbone.

Les projets de construction de lignes à grande vitesse sont l'exemple même d'une démarche axée prioritairement sur la création de nouvelles mobilités associée à la défense de l'environnement. Nul n'ignore que les grands chantiers de construction constituent autant de menaces pour l'équilibre des écosystèmes. Désormais il ne suffit plus de démontrer les avantages socioéconomiques d'un projet pour avoir accès à un financement national ou européen. II faut également mettre en avant toutes les démarches entreprises pour prévenir les risques environnementaux et éviter, réduire ou compenser les éventuels impacts négatifs.

II s'agit de démarches à multiples facettes. Toutes les actions doivent être mesurables en fonction de repères fixés de manière objective par des agences tierces. Parmi les cadres structurants servant à cette fin, citons l'exemple des "Lignes directrices pour le reporting en matière de développement durable ". Le document correspondant est le fruit d'une initiative, baptisée GRI ou Global Reporting Initiative, lancée vers la fin des années 1990 dans le but d'aider les sociétés commerciales et autres organisations dans la rédaction de rapports sur leurs performances, non seulement économiques et sociétales mais également environnementales(4).

La GRI propose une série d'indicateurs pour mesurer l'évolution, en matière de développement durable, des acteurs économiques. Dans leur préface, les auteurs expliquent que " le développement durable a pour but de répondre aux besoins du présent sans compromettre la

(3) Cf. dans le présent numéro l'article de Ginny Hill, "Le développement durable et le monde des grandes entreprises ", p. 41.

(4) La GRI, créée en 1997, est une initiative conjointe du Programme des Nations Unies pour l'environnement (PNUE) et de la Coalition pour des économies écologiquement responsables (CERES). 
capacité des générations futures à répondre aux leurs ". Pour atteindre cet objectif, il faut non seulement adapter ses comportements, mais également communiquer régulièrement et en toute transparence sur les actions engagées.

Le référentiel comporte un total de 79 indicateurs. II propose des définitions pour tous les concepts clés : contexte, exhaustivité, durabilité, périodicité... qui les sous-tendent. Les indicateurs se répartissent sur 7 catégories : économie, environnement, droits de l'homme, relations sociales et travail décent, responsabilité vis-à-vis des produits, société.

Pour le présent article, c'est la catégorie " environnement " qui revêt toute son importance. Sous cette rubrique, il s'agit de mesurer les impacts d'une entreprise ou d'une organisation sur les systèmes naturels (y compris les écosystèmes), ses performances dans la gestion de la chaîne de production et dans le respect de la biodiversité. Les facteurs utilisés dans l'évaluation de telles performances sont l'énergie, l'eau, la biodiversité, les émissions, effluents et déchets, les produits et services, le respect des textes, les transports. Inutile donc d'ajouter que le traducteur, quelle que soit sa spécialité, a de fortes chances de se retrouver confronté à des documents relatifs à l'une ou l'autre de ces nombreuses manifestations de ce que l'on appelle communément le " développement durable " !

Le texte de la Global Reporting Initiative existe en anglais et en français(5), ce qui peut être une aubaine pour les traducteurs travaillant dans cette combinaison linguistique. Un bémol cependant : la traduction française laisse parfois à désirer, elle est donc à manier avec précaution.

Pour revenir au domaine du transport, précisons que, selon des chiffres récents, $27 \%$ des émissions globales de gaz à effet de serre, toutes sources confondues, proviendraient de ce secteur, qui est également responsable de $31 \%$ de la consommation d'énergie finale (données CITEPA(6) 2007). Rien d'étonnant donc à ce que les différents acteurs se soient engagés dans des recherches de grande envergure pour améliorer leur performance et réduire leurs effets nocifs.

Dans le cas de notre exemple d'une ligne ferroviaire à grande vitesse, lorsqu'il s'agit de décider du tracé de la ligne, il faut non seulement prévoir des sondages archéologiques pour sauvegarder le patrimoine historique et conserver les restes du passé pour les générations à venir mais également se conformer aux exigences de la législation sur l'eau. Et cela ne s'arrête pas là. II faut aussi étudier la flore et la faune des zones concernées par le passage du futur tracé, surtout dans le cas de zones de protection spéciale (ZPS) créées en application de la directive européenne 79/409/CEE (plus connue sous le nom " directive oiseaux ") ou de la directive 92/43/CEE dite "directive habitats ", de zones naturelles d'intérêt écologique, faunistique et floristique (ZNIEFF) ou de celles faisant partie du réseau Natura 2000(7).

(5) www.globalreporting.org/resourcelibrary/French-G3-Reporting-Guidelines.pdf

(6) Centre interprofessionnel technique d'études de la pollution atmosphérique.

(7) http://www.developpement-durable.gouv.fr/-Natura-2000,2414-.html 
Ainsi, le prolongement du TGV Atlantique vers le sud doit traverser plusieurs zones riches en espèces vulnérables dont l'Outarde canepetière, oiseau dont la population est menacée d'extinction. Ces zones abritent également de très nombreuses autres espèces d'intérêt communautaire. La construction de la ligne pourrait donc perturber des populations d'oiseaux déjà fragilisées ou provoquer la destruction de leurs nichées.

Ces incidences directes cumulatives ont donc imposé de prendre des mesures efficaces destinées à éviter les conséquences néfastes, faute de quoi les autorisations nécessaires n'auraient jamais été accordées....

Qui parle de développement durable doit donc également tenir compte des exigences en matière de flore et de faune. Le traducteur avisé a tout intérêt à parfaire ses connaissances sur le sujet. Dans l'exemple donné on aborde le cas du chemin de fer mais les mêmes principes s'appliquent à la construction routière et à tous les travaux, quels qu'ils soient, dans des zones touchées de près ou de loin par les enjeux du développement durable. Un fabricant de papier qui emploie des produits chimiques dans son processus de fabrication n'a pas le droit de déverser ses eaux usées dans les ruisseaux ou les rivières qui passent à côté de ses usines...

Désormais, le développement durable, au sens le plus large du terme, touche tous les aspects de notre vie. Nul ne peut ignorer cette évolution aujourd'hui résolument en marche. Outre le secteur du transport, d'autres domaines sont marqués par la même empreinte : scientifique (recherches liées à la transition énergétique), agriculture (engrais, désherbants), bâtiment (techniques de construction écologiques), juridique (législations nationale et européenne) : la liste est longue et loin d'être exhaustive.

Pour le traducteur indépendant, exercer son métier sans entrer en contact de près ou de loin avec ce thème relève désormais de l'exploit. Le vocabulaire se précise et si les puristes ne sont pas toujours d'accord avec certaines expressions entrées dans le vocabulaire courant, force est d'admettre qu'elles ont au moins le mérite d'exister. L'émergence du concept de "développement durable " et ses conséquences pour notre profession sont indéniables. II s'agit d'un beau défi que nous nous devons de relever, non seulement dans notre intérêt professionnel mais également dans l'intérêt des générations à venir.

cross.christine@gmail.com 